\title{
Kontaktallergien und Krebs
}

\author{
Ungefähr 20\% der Europäer sind \\ allergisch gegen eine oder mehrere \\ Substanzen des europäischen Patch- \\ Test-Panels. Während bisher viele \\ Studien die Beziehungen zwischen \\ Allergien Typ 1 und Krebs unter- \\ suchten, suchten nur wenige nach \\ den Beziehungen zwischen Krebs \\ und einer Typ-IV-Allergie.
}

— Forscher der Universität Kopenhagen haben jetzt die mögliche Beziehung zwischen Kontaktallergie Typ IV und Krebs untersucht. Für ihre Studie konnten sie die Daten zweier Register heranziehen: ein Krankenhaus-Register mit Dermatitis-Patienten, getestet zwischen 1984 und $2008(\mathrm{n}=17000)$ und das landesweite dänische Krebsregister. 6065 (35\%) Patienten waren mindestens gegen ein Allergen positiv. Frauen (41\%) waren reaktiver als Männer (26\%). Die logistischen Regressionsanalysen wurden mit mehr als 40 Krebssubtypen durchgeführt.

Eine inverse Beziehung wurde für beide Geschlechter zwischen Kontaktallergie und Nicht-Melanom-Hautkrebs bzw. Brustkrebs identifiziert. Ein umgekehrter Trend wurde bei Frauen mit Kontaktallergie und Gehirntumoren gefunden. Positiv hingegen war die Beziehung zwischen Kontaktallergie und Harnblasenkrebs.

\section{Kommentar}

Die umgekehrten Assoziationen unterstützen die Hypothese, dass Personen mit einer Allergie Typ IV weniger Krebs haben, weil ihr Immunsystem gegen Krebszellen getriggert ist. Dass bei den Allergikern vermehrt Harnblasenkrebs gefunden wird, könnte durch die Akkumulation von Metaboliten in der Blase begründet sein.

K. MALBERG =

\section{Hier muss der Dummy durch eine Anzeige ersetzt werden !!}

